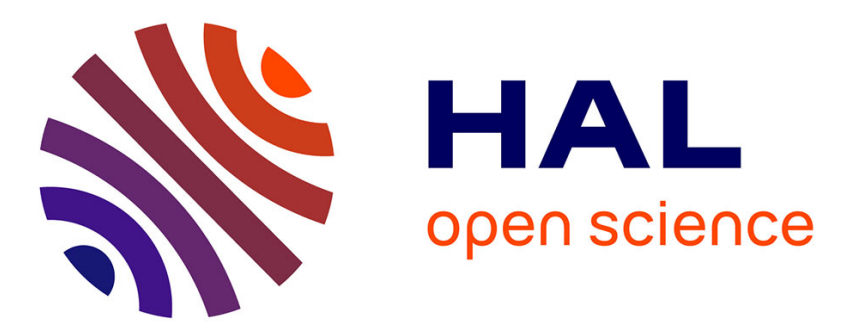

\title{
Decomposing degenerate graphs into locally irregular subgraphs
}

\author{
Julien Bensmail, François Dross, Nicolas Nisse
}

\section{To cite this version:}

Julien Bensmail, François Dross, Nicolas Nisse. Decomposing degenerate graphs into locally irregular subgraphs. Graphs and Combinatorics, 2020, 36 (6), pp.1869-1889. 10.1007/s00373-020-02193-6 . hal-02090804v3

\section{HAL Id: hal-02090804 \\ https://hal.science/hal-02090804v3}

Submitted on 10 Apr 2020

HAL is a multi-disciplinary open access archive for the deposit and dissemination of scientific research documents, whether they are published or not. The documents may come from teaching and research institutions in France or abroad, or from public or private research centers.
L'archive ouverte pluridisciplinaire HAL, est destinée au dépôt et à la diffusion de documents scientifiques de niveau recherche, publiés ou non, émanant des établissements d'enseignement et de recherche français ou étrangers, des laboratoires publics ou privés. 


\title{
Decomposing degenerate graphs into locally irregular subgraphs ${ }^{1}$
}

\author{
Julien Bensmail ${ }^{\mathrm{a}}$, François Dross ${ }^{\mathrm{a}}$, Nicolas Nisse ${ }^{\mathrm{a}}$ \\ ${ }^{a}$ Université Côte d'Azur, CNRS, Inria, I3S, France
}

\begin{abstract}
A (undirected) graph is locally irregular if no two of its adjacent vertices have the same degree. A decomposition of a graph $G$ into $k$ locally irregular subgraphs is a partition $E_{1}, \ldots, E_{k}$ of $E(G)$ into $k$ parts each of which induces a locally irregular subgraph. Not all graphs decompose into locally irregular subgraphs; however, it was conjectured that, whenever a graph does, it should admit such a decomposition into at most three locally irregular subgraphs. This conjecture was verified for a few graph classes in recent years.

This work is dedicated to the decomposability of degenerate graphs with low degeneracy. Our main result is that decomposable $k$-degenerate graphs decompose into at most $3 k+1$ locally irregular subgraphs, which improves on previous results whenever $k \leq 9$. We improve this result further for some specific classes of degenerate graphs, such as bipartite cacti, $k$-trees, and planar graphs. Although our results provide only little progress towards the leading conjecture above, the main contribution of this work is rather the decomposition schemes and methods we introduce to prove these results.
\end{abstract}

Keywords: locally irregular decompositions; degenerate graphs; cacti; $k$-trees; planar graphs.

\section{Introduction}

A graph $G$ is locally irregular if no two of its adjacent vertices have the same degree, i.e., for every edge $u v \in E(G)$ we have $d(u) \neq d(v)$. In general, $G$ might be far from being locally irregular (e.g. when $G$ is regular), and we might then be interested in decomposing $G$ into locally irregular subgraphs. The term "decomposition" is, throughout this paper, understood as an edge-partition. A locally irregular decomposition of $G$ is then a partition of $E(G)$ into parts $E_{1}, \ldots, E_{k}$ each of which induces a locally irregular subgraph. The least number $k$ such that $G$ can be decomposed into $k$ locally irregular subgraphs is called the irregular chromatic index of $G$, and is denoted $\chi_{\text {irr }}^{\prime}(G)$. There are contexts where $G$ might admit no locally irregular decomposition at all (consider e.g. any odd-length path), in which case we define $\chi_{\text {irr }}^{\prime}(G)=\infty$. We call $G$ decomposable if $\chi_{\text {irr }}^{\prime}(G)$ is finite, while we call $G$ exceptional otherwise.

Locally irregular decompositions were introduced by Baudon, Bensmail, Przybyło and Woźniak [2] as a tool to deal with some cases of the well-known 1-2-3 Conjecture posed by Karonski, Łuczak and Thomason [6]. The main open question on locally irregular decompositions is about whether every decomposable graph has a decomposition into three locally irregular subgraphs:

Conjecture 1.1 (Baudon, Bensmail, Przybyło, Woźniak [2]). For every decomposable graph G, we have $\chi_{\mathrm{irr}}^{\prime}(G) \leq 3$.

For a better understanding of Conjecture 1.1, it is worth mentioning that exceptional graphs comprise exactly three families $\mathcal{P}, \mathcal{C}, \mathcal{T}$ of graphs. The family $\mathcal{P}$ is the one of all odd-length paths, while $\mathcal{C}$ is the family of all odd-length cycles. The definition of $\mathcal{T}$ is recursive:

1. The triangle $K_{3}$ belongs to $\mathcal{T}$.

\footnotetext{
${ }^{1}$ This work has been supported by the French government, through the UCA JEDI Investments in the Future project managed by the National Research Agency (ANR) with the reference number ANR-15-IDEX-01, by the ANR project Digraphs with the reference number ANR-19-CE48-0013, and by the STIC-AmSud Program with the project GALOP.
} 
2. Every other graph in $\mathcal{T}$ can be constructed by 1) taking an auxiliary graph $H$ being either an even-length path or an odd-length path with a triangle glued to one of its ends, then 2) choosing a graph $G \in \mathcal{T}$ containing a triangle with at least one vertex, say $v$, of degree 2 in $G$, and finally 3) identifying $v$ with a vertex of degree 1 of $H$.

In other words, the graphs in $\mathcal{T}$ consist of disjoint triangles connected in a tree-like fashion, such that, when contracting the triangles, two triangle vertices are joined by an odd-length path, while a triangle vertex and an original degree-1 vertex are joined by an even-length path.

Theorem 1.2 (Baudon, Bensmail, Przybyło, Woźniak [2]). A connected graph is exceptional if and only if it belongs to $\mathcal{P} \cup \mathcal{C} \cup \mathcal{T}$.

Regarding our investigations in this paper, it is worth emphasizing that all exceptional graphs have odd size, maximum degree at most 3, low degeneracy (at most 2), and are planar. Recall that a graph $G$ is called $k$-degenerate if $|V(G)| \leq k+1$ or there exists $v \in V(G)$ with degree at most $k$ such that $G-v$ is $k$-degenerate.

Conjecture 1.1 was proved for decomposable trees, complete graphs, complete bipartite graphs, some Cartesian products of graphs, regular graphs with degree at least $10^{7}$ [2], graphs with minimum degree at least $10^{10}$ [9], and decomposable split graphs [7]. For some classes of graphs, there is still some gap between the bound in Conjecture 1.1 and the best known one on the irregular chromatic index. In [4], decomposable bipartite graphs were proved to have irregular chromatic index at most 10, which was improved down to 7 in [8]. In [1], decomposable graphs with maximum degree 3 were proved to have irregular chromatic index at most 5 , which was improved down to 4 in $[8]$.

A first constant upper bound of 328 on the irregular chromatic index of decomposable graphs was given in [4] by Bensmail, Merker and Thomassen. The steps of the proof can be roughly sketched as follows:

- In every decomposable graph $G$, one can find a locally irregular subgraph whose removal leaves a graph whose all connected components are of even size (number of edges), thus decomposable. Thus, towards Conjecture 1.1, one can actually focus on graphs with even size, the price for this being one additional graph in a decomposition.

Theorem 1.3 (Bensmail, Merker, Thomassen [4]). Let $\mathcal{G}$ be a hereditary family of connected graphs. Then, $\max \left\{\chi_{\mathrm{irr}}^{\prime}(G): G \in \mathcal{G}\right.$ is decomposable $\} \leq \max \left\{\chi_{\mathrm{irr}}^{\prime}(G): G \in \mathcal{G}\right.$ has even size $\}+1$.

- Once $G$ has even size, it can then be decomposed into two graphs $D$ and $H$, where $D$ is $\left(2 \cdot 10^{10}+2\right)$-degenerate and has all of its connected components of even size, while $H$ is of minimum degree at least $10^{10}$. These two graphs can be further decomposed as follows:

- $H$, because of its large minimum degree, can be decomposed into at most three locally irregular subgraphs, according to a result of Przybyło [9].

- $D$ can be decomposed into at most $\left\lceil\log _{2}\left(2 \cdot 10^{10}+3\right)\right\rceil+1$ bipartite graphs with even size, which can each be decomposed into at most nine locally irregular subgraphs.

As Lužar, Przybyło and Soták proved that bipartite graphs with even size can even be decomposed into at most six locally irregular subgraphs [8], the exact same proof yields that every decomposable graph has irregular chromatic index at most 220. More precisely, this improved bound is a consequence of the following:

Theorem 1.4 ([4, 8]). For every connected $k$-degenerate graph $G$ with even size, we have

$$
\chi_{\mathrm{irr}}^{\prime}(G) \leq 6 \cdot\left(\left\lceil\log _{2}(k+1)\right\rceil+1\right) .
$$

Consequently, by Theorem 1.3, for every decomposable $k$-degenerate graph $G$ we have

$$
\chi_{\text {irr }}^{\prime}(G) \leq 6 \cdot\left(\left\lceil\log _{2}(k+1)\right\rceil+1\right)+1 .
$$


Our results. As a main result in this work, we give, in Section 3, a new upper bound on the irregular chromatic index of graphs with given degeneracy:

Theorem 1.5. For every connected $k$-degenerate graph $G$ with even size, we have $\chi_{\mathrm{irr}}^{\prime}(G) \leq 3 k$. Consequently, by Theorem 1.3, for every decomposable $k$-degenerate graph $G$ we have $\chi_{\mathrm{irr}}^{\prime}(G) \leq$ $3 k+1$.

Theorem 1.5 improves Theorem 1.4 for graphs with low degeneracy. More precisely, $3 k<$ $6 \cdot\left(\left\lceil\log _{2}(k+1)\right\rceil+1\right)$ whenever $k \leq 9$. As notable cases, we get that decomposable 2-degenerate graphs (which include outerplanar graphs, series-parallel graphs, etc.) have irregular chromatic index at most 7 (versus 19 previously), and decomposable planar graphs, which are 5-degenerate, have irregular chromatic index at most 16 (versus 25 previously). Although these bounds are only a little step towards Conjecture 1.1, the main interest of our contribution is in the decomposition methods and strategies we develop to prove these results. An important point also is that most of the graph classes we consider actually include exceptional graphs, which are one of the reasons why Conjecture 1.1 seems tricky to prove for these classes.

In general, the bound in Theorem 1.5 remains distant from the one conjectured in Conjecture 1.1. As a side aspect in this paper, we also focus on improving Theorem 1.5 for particular classes of degenerate graphs. In Section 2, we prove a key ingredient behind our proof of Theorem 1.5, which is that decomposable bipartite cacti verify Conjecture 1.1. In Section 4, we improve Theorem 1.5 for various classes of degenerate graphs, namely decomposable $k$-trees (for which we prove $2 k$ ) and decomposable planar graphs (for which we prove 15). Conclusions and perspectives for further works are gathered in Section 5.

\section{Decomposing good cacti into locally irregular subgraphs}

Throughout this paper, for a graph $G$ and a given subset $F \subseteq E(G)$ (resp., $X \subseteq V(G)$ ), we denote by $G[F]$ (resp., $G[X]$ ) the subgraph of $G$ induced by $F$ (resp., by $X$ ).

The proof of our main result, Theorem 1.5, relies of the fact that $k$-degenerate graphs can, in general, be decomposed into a certain number of particular cacti. Recall that a cactus is a graph in which no two cycles intersect in more than one vertex. The cacti we are interested in are those that are bipartite (i.e., which have no odd-length cycle) and have no connected component being an odd-length path (as graphs having such are not decomposable, recall Theorem 1.2). Such cacti we call good cacti for convenience.

By Theorem 1.2, note that $\chi_{\mathrm{irr}}^{\prime}(G)$ is defined for every good cactus $G$. Furthermore, there exist infinitely many good cacti $G$ verifying $\chi_{\text {irr }}^{\prime}(G)=3$. Any cycle with length congruent to 2 modulo 4 is an example. Actually, it is worth recalling that even infinitely many trees with irregular chromatic index 3 exist, as reported in [3]. Still, we prove that Conjecture 1.1 holds for good cacti.

Before proving this claim, let us give a few more details on the case of trees, as some of these will be mentioned in our proofs. In [3], Baudon, Bensmail and Sopena gave a description of trees with irregular chromatic index exactly 3, through the notion of shrub. A shrub is a tree being rooted at a root vertex $r$ with degree 1 . The authors proved that shrubs all admit "almost" locally irregular decompositions (being locally irregular decompositions where only the root is allowed to belong to a connected component that is not locally irregular) into at most two parts. Now, when considering a general tree $T$ with a vertex $v$ of degree $d \geq 2$, we can see $T$ as $d$ shrubs whose roots were identified into $v$. The authors proved that, except in very particular cases, almost locally irregular decompositions into at most two parts of these shrubs, when combined, can yield one locally irregular decomposition into at most two parts of $T$. More precisely, a tree with irregular chromatic index 3 must be constructed using bad shrubs, whose all almost locally irregular decompositions into at most two parts are very particular.

From the investigations in [3], one can in particular deduce the following:

Theorem 2.1 (Baudon, Bensmail, Sopena [3]). Let $T$ be a tree with $\chi_{\mathrm{irr}}^{\prime}(T)=3$. Then:

- $|E(T)|$ is necessarily odd;

- $T$ has no degree-3 or degree-4 vertex adjacent to a leaf. 
We are now ready for proving our main result in this section.

Theorem 2.2. For every good cactus $G$, we have $\chi_{\text {irr }}^{\prime}(G) \leq 3$.

Proof. Clearly, we may assume that $G$ is connected. The proof is by induction on the number of edges of $G$. The base cases are the path of length 2 and, more generally, any tree that is an even-length path or any bipartite cycle, in which cases the result holds [2, 3]. Hence, we may assume that $G$ contains at least one cycle and that $G$ is not reduced to this cycle.

Let $v \in V(G)$ be a vertex of $G$ that belongs to a single cycle $C$. Let us consider the (unique) connected component $X$ containing $v$ after the removal of the two edges of $C$ that are incident to $v$. If $X$ is a tree (not reduced to the single vertex $v$ ), then, in what follows, we denote it by $T_{v}$ and refer to it as the tree pending in $v$. Similarly, let $v \in V(G)$ be a vertex of $G$ that is not contained in any cycle. If there is an edge incident to $v$ whose removal makes the connected component containing $v$ to be a tree (with at least one edge), then we denote by $T_{v}$ this connected component and regard it as the tree pending at $v$.

First, we prove that we can assume that all the pending trees of $G$ have a simple form. Here, a spider with degree $d \geq 1$ is any graph obtained from a star whose center has degree $d$ by subdividing each edge at most once. That is, a spider is obtained by identifying one end of some paths of length at most 2. Note that every spider is locally irregular unless it is a path $P_{i}$ with length $i \in\{1,3,4\}$.

- Let us first assume that there exists $u \in V(G)$ whose pending tree $T_{u}$ is a path $(u, v, w, x)$ with three edges such that $u$ is one end of this path. Then $G^{\prime}=G[V(G) \backslash\{w, x\}]$ is a good cactus (and not an odd-length path) and $\left|E\left(G^{\prime}\right)\right|<|E(G)|$, and, by the induction hypothesis, $G^{\prime}$ can be decomposed into at most three locally irregular subgraphs $G_{1}^{\prime}, G_{2}^{\prime}, G_{3}^{\prime}$. W.l.o.g., $u v \in E\left(G_{1}^{\prime}\right)$. Then, let $G_{2}^{*}$ be induced by $G_{2}^{\prime}$ and the edges $v w$ and $w x$ (i.e., $G_{2}^{*}$ is the disjoint union of a locally irregular graph $G_{2}^{\prime}$ and of a path $(v, w, x)$ of length 2 , i.e., $G_{2}^{*}$ is locally irregular). Then, $G_{1}^{\prime}, G_{2}^{*}, G_{3}^{\prime}$ are three locally irregular subgraphs decomposing $G$, i.e., $\chi_{\text {irr }}^{\prime}(G) \leq 3$.

- Second, let us assume that there exists $v \in V(G)$ whose pending tree $T_{v}$ is a path $P_{3}=$ $(u, v, w, x)$ of length 3 such that $v$ is not an end of this path. Then, $G^{\prime}=G[V(G) \backslash\{w, x\}]$ is a good cactus (because $G^{\prime}$ still contains a cycle and so cannot be an odd-length path) and $\left|E\left(G^{\prime}\right)\right|<|E(G)|$. Moreover, $v$ has degree at most 3 in $G^{\prime}$ (by definition of a pending tree); let thus $a, b$ denote its at most two neighbours distinct from $u$.

By the induction hypothesis, $G^{\prime}$ can be decomposed into at most three locally irregular subgraphs $G_{1}^{\prime}, G_{2}^{\prime}, G_{3}^{\prime}$. W.l.o.g., $u v \in E\left(G_{1}^{\prime}\right)$. Then, either $a v$ or bv (or both) are in $E\left(G_{1}^{\prime}\right)$ (otherwise, $u v$ would be an isolated edge in $G_{1}^{\prime}$, contradicting that it is locally irregular). W.l.o.g., say $a v \in E\left(G_{1}^{\prime}\right)$. Moreover, if $b v \notin E\left(G_{1}^{\prime}\right)$, then say, w.l.o.g., that $b v \in E\left(G_{2}^{\prime}\right)$. Then, let $G_{3}^{*}$ be induced by $G_{3}^{\prime}$ and the edges $w v$ and $x w$ (i.e., $G_{3}^{*}$ is the disjoint union of a locally irregular graph $G_{3}^{\prime}$ and of a path $(v, w, x)$ of length 2 , i.e., $G_{3}^{*}$ is locally irregular). Then, $G_{1}^{\prime}, G_{2}^{\prime}, G_{3}^{*}$ are three locally irregular subgraphs decomposing $G$, i.e., $\chi_{\text {irr }}^{\prime}(G) \leq 3$.

- Third, let us assume that there exists a vertex $v \in V(G)$, not in any cycle, with a pending tree which is a path $P_{4}=(a, b, v, c, d)$ of length 4 where $v$ is the center. Then, $G^{\prime}=$ $G\left[E(G) \backslash E\left(T_{v}\right)\right]$ is a good cactus (because $G^{\prime}$ still contains a cycle and so cannot be an odd-length path) and $\left|E\left(G^{\prime}\right)\right|<|E(G)|$. Note that $v$ has degree 1 in $G^{\prime}$.

By the induction hypothesis, $G^{\prime}$ can be partitioned into at most three locally irregular subgraphs $G_{1}^{\prime}, G_{2}^{\prime}, G_{3}^{\prime}$. W.l.o.g., the edge incident to $v$ in $G^{\prime}$ belongs to $G_{1}^{\prime}$. Then, let $G_{2}^{*}$ (resp., $G_{3}^{*}$ ) be induced by $E\left(G_{2}^{\prime}\right)$ and $\{a b, b v\}$ (resp., induced by $E\left(G_{3}^{\prime}\right)$ and $\{v c, c d\}$ ). Then, $G_{1}^{\prime}, G_{2}^{*}, G_{3}^{*}$ are three locally irregular subgraphs decomposing $G$, i.e., $\chi_{\text {irr }}^{\prime}(G) \leq 3$.

- Finally, let us assume that there exists some vertex $v \in V(G)$ with pending tree $T_{v}$ that is a spider distinct from $P_{1}, P_{3}, P_{4}$. As mentioned above, $T_{v}$ is locally irregular. Then, $G^{\prime}=G\left[E(G) \backslash E\left(T_{v}\right)\right]$ is a good cactus (and not an odd-length path) and $\left|E\left(G^{\prime}\right)\right|<|E(G)|$. Moreover, $v$ has degree at most 2 in $G^{\prime}$ (by definition of a pending tree) and let $a, b$ be its at most two neighbours in $G^{\prime}$. By the induction hypothesis, $G^{\prime}$ can be decomposed into at most three locally irregular subgraphs $G_{1}^{\prime}, G_{2}^{\prime}, G_{3}^{\prime}$. W.l.o.g., $\{v a, v b\} \subseteq E\left(G_{1}^{\prime}\right) \cup E\left(G_{2}^{\prime}\right)$. Then, 
let $G_{3}^{*}$ be induced by $G_{3}$ and the edges in $E\left(T_{v}\right)$ (so $G_{3}^{*}$ is the disjoint union of two locally irregular graphs). Then, $G_{1}^{\prime}, G_{2}^{\prime}, G_{3}^{*}$ are three locally irregular subgraphs decomposing $G$, i.e., $\chi_{\text {irr }}^{\prime}(G) \leq 3$.

From the previous reduction rules, we may now assume that, for every pending tree $T_{v}, v$ belongs to a cycle and $T_{v}$ is either a single edge or a length- 4 path $P_{4}$ with $v$ as central vertex. Indeed, otherwise, one of the previous four cases would apply: if $v$ is the single vertex with degree at least 3 in $T_{v}$, then the result is obvious; while, otherwise, for $w$ being a vertex of $T_{v}$ with degree at least 3 and as far as possible from $v$, the pending tree $T_{w}$ is a pending tree to which one of the previous four rules applies.

We are now ready to conclude. If $G$ contains a single cycle, then let $C$ denote this cycle and let $u^{*} \in V(C)$ be any vertex of $C$ with degree at least 3 (recall that we have assumed that $G$ is not only a cycle). Otherwise, let $C, C^{\prime}$ be two cycles of $G$ maximising the distance between them, and let $u^{*} \in V(C)$ be the (unique) vertex of $C$ that is the closest to $C^{\prime}$ (hence, all vertices of $C$ except possibly $u^{*}$ belong to a single cycle). Note that, because $G$ is a good cactus, $C$ has even size at least 4, so some $v^{*} \in V(C) \backslash N\left[u^{*}\right]$ exists and let $a$ and $b$ the two neighbours of $v^{*}$ in $C$. Note also that $a, b$ and $v^{*}$ belong to a single cycle. There are several cases to be considered.

- Case 1: $v^{*}$ has degree 2 in $G$ (i.e., $v^{*}$ has no pending tree).

If one, say $a$, of $a, b$, has a pending tree $T_{a}$, then let $X=\left\{a v^{*}\right\} \cup E\left(T_{a}\right)$ and note that $X$ induces a locally irregular graph. Recall that $T_{a}$ either has a single edge or is a path of length 4 centered at $a$. Then, $G^{\prime}=G[E(G) \backslash X]$ is a good cactus (not an odd-length path since $u^{*}$ has degree at least 3). By the induction hypothesis, $G^{\prime}$ can be decomposed into at most three locally irregular subgraphs $G_{1}^{\prime}, G_{2}^{\prime}, G_{3}^{\prime}$. Note that $a$ and $v^{*}$ have degree 1 in $G^{\prime}$, so we may assume that they are not incident to any edge in $G_{1}^{\prime}$. Then, let $G_{1}^{*}$ be induced by $G_{1}$ and the edges in $X$ (so $G_{1}^{*}$ is the disjoint union of two locally irregular graphs). Then, $G_{1}^{*}, G_{2}^{\prime}, G_{3}^{\prime}$ are three irregular subgraphs decomposing $G$, i.e., $\chi_{\text {irr }}^{\prime}(G) \leq 3$.

So we can assume that both $a$ and $b$ have degree 2, in which case we can define $X$ as the set of (two) edges incident to $v^{*}$, and proceed as in the previous case.

- Case 2: $v^{*}$ has degree 3 in $G$ (i.e., $v^{*}$ has $P_{1}$ as pending tree).

Assume not both pending trees $T_{a}$ and $T_{b}$ (if they exist) are $P_{1}$, say $T_{a}$ is not $P_{1}$ (i.e., $T_{a}$ is either empty or $\left.P_{4}\right)$. Let $X=\left\{a v^{*}\right\} \cup E\left(T_{v}\right) \cup E\left(T_{a}\right)$ and note that $X$ induces a locally irregular graph. Then, $G^{\prime}=G[E(G) \backslash X]$ is a good cactus (not an odd-length path since $u^{*}$ has degree at least 3). By the induction hypothesis, $G^{\prime}$ can be decomposed into at most three locally irregular subgraphs $G_{1}^{\prime}, G_{2}^{\prime}, G_{3}^{\prime}$. Note that $a$ and $v^{*}$ have degree 1 in $G^{\prime}$, so we may assume that they are not incident to any edge in $G_{1}^{\prime}$. Then, let $G_{1}^{*}$ be induced by $G_{1}$ and the edges in $X$ (so $G_{1}^{*}$ is the disjoint union of two locally irregular graphs). Then, $G_{1}^{*}, G_{2}^{\prime}, G_{3}^{\prime}$ are three irregular subgraphs decomposing $G$, i.e., $\chi_{\text {irr }}^{\prime}(G) \leq 3$.

So we may assume that both $T_{a}$ and $T_{b}$ are $P_{1}$, in which case we set $X=\left\{a v^{*}, b v^{*}\right\} \cup E\left(T_{v^{*}}\right)$ which induces a locally irregular graph. Then, $G^{\prime}=G[E(G) \backslash X]$ is a good cactus (not an odd-length path since $u^{*}$ has degree at least 3). By the induction hypothesis, $G^{\prime}$ can be decomposed into at most three irregular subgraphs $G_{1}^{\prime}, G_{2}^{\prime}, G_{3}^{\prime}$. Note that $a$ and $b$ have degree 2 in $G^{\prime}$. However, both edges incident to $a$ (resp., to $b$ ) must belong to the same part of the decomposition (as otherwise $T_{a}$, resp., $T_{b}$, would induce an isolated edge). Therefore, we may assume that they are not incident to any edge in $G_{1}^{\prime}$. Then, let $G_{1}^{*}$ be induced by $G_{1}$ and the edges in $X$ (so $G_{1}^{*}$ is the disjoint union of two locally irregular graphs). Then, $G_{1}^{*}, G_{2}^{\prime}, G_{3}^{\prime}$ are three locally irregular subgraphs decomposing $G$, i.e., $\chi_{\text {irr }}^{\prime}(G) \leq 3$.

- Case 3: $v^{*}$ has degree 4 in $G$ (i.e., $v^{*}$ is the center of a $P_{4}$ as pending tree).

Assume not both $T_{a}$ and $T_{b}$ are $P_{4}$, say $T_{a}$ is not a $P_{4}$; then let $X=\left\{a v^{*}\right\} \cup E\left(T_{v}\right) \cup E\left(T_{a}\right)$ and note that $X$ induces a locally irregular graph. Then, $G^{\prime}=G[E(G) \backslash X]$ is a good cactus (not an odd-length path since $u^{*}$ has degree at least 3). By the induction hypothesis, $G^{\prime}$ can be decomposed into at most three irregular subgraphs $G_{1}^{\prime}, G_{2}^{\prime}, G_{3}^{\prime}$. Note that $a$ and $v^{*}$ have degree 1 in $G^{\prime}$, so we may assume that they are not incident to any edge in $G_{1}^{\prime}$. Then, 
let $G_{1}^{*}$ be induced by $G_{1}$ and the edges in $X$ (so $G_{1}^{*}$ is the disjoint union of two locally irregular graphs). Then, $G_{1}^{*}, G_{2}^{\prime}, G_{3}^{\prime}$ are three locally irregular subgraphs decomposing $G$, i.e., $\chi_{\text {irr }}^{\prime}(G) \leq 3$.

The last case is when all of $v^{*}, a, b$ have degree 4 , i.e., each of these vertices has two pending paths of length 2 attached. In that case, let $X=\left\{a v^{*}, b v^{*}\right\} \cup E\left(T_{a}\right) \cup E\left(T_{b}\right) \cup E\left(T_{v^{*}}\right)$. Note that this time the subgraph induced by $X$ is not locally irregular.

Then, $G^{\prime}=G[E(G) \backslash X]$ is a good cactus (not an odd-length path since $u^{*}$ has degree at least 3). By the induction hypothesis, $G^{\prime}$ can be decomposed into at most three irregular subgraphs $G_{1}^{\prime}, G_{2}^{\prime}, G_{3}^{\prime}$. Note that $a$ and $b$ have degree 1 in $G^{\prime}$. Say the edge $a^{\prime} a$ incident to $a$ in $G^{\prime}$ belongs to $G_{1}^{\prime}$ and let $d$ be the degree of $a^{\prime}$ in $G_{1}^{\prime}$. Also, w.l.o.g., say that the edge incident to $b$ in $G^{\prime}$ is not in $G_{2}^{\prime}$. It remains two cases to be considered.

- If $d \neq 4$, then let $G_{1}^{*}$ be the subgraph induced by the edges of $G_{1}^{\prime}$ plus $\left\{a v^{*}\right\} \cup E\left(T_{a}\right) \cup$ $E\left(T_{v^{*}}\right)$ (it is a locally irregular graph) and let $G_{2}^{*}$ be induced by $G_{2}^{\prime}$ and $E\left(T_{b}\right) \cup\left\{b v^{*}\right\}$ (also locally irregular). Then $G_{1}^{*}, G_{2}^{*}, G_{3}^{\prime}$ is the desired solution.

- If $d=4$, then let $G_{1}^{*}$ be the subgraph induced by the edges of $G_{1}^{\prime}$ plus $E\left(T_{a}\right)$ (it is a locally irregular graph) and let $G_{2}^{*}$ be induced by $G_{2}^{\prime}$ and $E\left(T_{v^{*}}\right) \cup E\left(T_{b}\right) \cup\left\{b v^{*}, a v^{*}\right\}$ (also locally irregular). Then $G_{1}^{*}, G_{2}^{*}, G_{3}^{\prime}$ is the desired solution.

\section{Decomposing degenerate graphs into good cacti}

In this section, we prove the first part of Theorem 1.5, hence the whole statement. Our proof relies on the following key lemma.

Lemma 3.1. Let $G$ be a graph, and let $\left(T_{1}, T_{2}\right)$ be a partial decomposition of $G$ into two good cacti. Consider a vertex $v$ of $G$ belonging to none of $T_{1}, T_{2}$. Then, for every two edges vu, vw incident to $v$, there exists a partial decomposition $\left(T_{1}^{*}, T_{2}^{*}\right)$ of $G$ into two good cacti, where $E\left(T_{1}^{*}\right) \cup E\left(T_{2}^{*}\right)=$ $E\left(T_{1}\right) \cup E\left(T_{2}\right) \cup\{v u, v w\}$.

Proof. Roughly, $T_{1}^{*}$ and $T_{2}^{*}$ will be obtained starting from $T_{1}$ and $T_{2}$, possibly switching edges from one of the subgraphs to the second graph, and adding each of $v u$ and $v w$ to one of the subgraphs. We need to consider a few cases. First let us assume that there exists some of $T_{1}$ and $T_{2}$, say $T_{1}$, such that $u$ and $w$ do not belong to the same connected component of the subgraph induced by $E\left(T_{1}\right)$ (possibly, $u$ or $w$ or both are not adjacent to any edge of $E\left(T_{1}\right)$ ). In that case, let $T_{2}^{*}=T_{2}$ and let $T_{1}^{*}$ be induced by $E\left(T_{1}\right) \cup\{v u, v w\}$. Clearly, adding these two edges to $T_{1}$ cannot create an odd-length cycle (since $u$ and $w$ are not in a same connected component of $T_{1}$ ) and it cannot create an odd-length path (since $T_{1}$ is a good cactus). Hence, $T_{1}^{*}$ is a good cactus and we are done.

Second, let us assume that $u$ and $w$ belong to a same connected component, say $C_{1}$ (resp., $C_{2}$ ), of $T_{1}$ (resp., of $T_{2}$ ).

- Let us first assume that, for $u^{\prime}, w^{\prime} \in\{u, w\}, u^{\prime} \neq w^{\prime}$, and for $x, y \in\{1,2\}, x \neq y$, we have:

- either $u^{\prime}$ has degree 1 in $C_{x}$ and $C_{x}$ has at least one vertex of degree at least 3 , or $u^{\prime}$ has degree at least 2 in $C_{x}$, and

- either $w^{\prime}$ has degree 1 in $C_{y}$ and $C_{y}$ has at least one vertex of degree at least 3 , or $w^{\prime}$ has degree at least 2 in $C_{y}$, and

In this case, let $T_{x}^{*}$ (resp. $T_{y}^{*}$ ) be obtained by adding $u^{\prime} v$ to $T_{x}$ (resp., by adding $w^{\prime} v$ to $T_{y}$ ). It is easy to see that $T_{1}^{*}$ and $T_{2}^{*}$ are good cacti since no odd-length cycles are created and $C_{i}$ (for both $i \in\{1,2\}$ ) cannot become an odd-length path.

- Second, let us assume that, for some of $C_{1}$ or $C_{2}$, w.l.o.g., say $C_{1}$, the subpath of $C_{1}$ from $u$ to $w$ has even length. In that case, let $T_{2}^{*}=T_{2}$ and let $T_{1}^{*}$ be induced by $E\left(T_{1}\right) \cup\{v u, v w\}$. Clearly, adding these two edges to $T_{1}$ cannot create an odd-length cycle and it cannot create an odd-length path (actually, $C_{1}$ becomes an even-length cycle plus, possibly, a pending path). Hence, $T_{1}^{*}$ is a good cactus and we are done. 
- If none of the previous cases holds, it must be that $C_{1}$ and $C_{2}$ are even-length paths with a common end in $\{u, w\}$, w.l.o.g., say $w$, and such that $u$ has degree 2 in both $C_{1}$ and $C_{2}$. Again, several cases must be considered. For $i \in\{1,2\}$, let $x_{i} \in V$ be the end (distinct from $w$ ) of $C_{i}$ and let $C_{i}^{b}$ (resp., $C_{i}^{a}$ ) be the subpath of $C_{i}$ between $x_{i}$ and $u$ (resp., between $u$ and $w)$. Moreover, $C_{i}^{a}$ and $C_{i}^{b}(i \in\{1,2\})$ have odd length (since $C_{i}$ has even length and the case when some of $C_{1}^{a}$ or $C_{2}^{a}$ has even length has been considered in the previous item).

- If $C_{1}^{a}$ is just the edge $u w$ (the case when $E\left(C_{2}^{a}\right)=u w$ is symmetric), then let $C_{1}^{*}$ be induced by the edges in $\left(E\left(C_{1}\right) \backslash\{u w\}\right) \cup\{u v\}$ (hence $C_{1}^{*}$ is an even-length path) and let $C_{2}^{*}$ be induced by the edges in $E\left(C_{2}\right) \cup\{w v, u w\}$ (hence, $C_{2}^{*}$ induces an even-length cycle, since $C_{2}^{a}$ is an odd-length path, plus one pending path, $C_{2}^{b}$, and one pending edge $w v)$. Then $T_{1}^{*}=\left(T_{1} \backslash C_{1}\right) \cup C_{1}^{*}$ and $T_{2}^{*}=\left(T_{2} \backslash C_{2}\right) \cup C_{2}^{*}$ are good cacti.

- Now, let us assume that $C_{1}^{a}$ and $C_{2}^{a}$ are not single edges. For $i \in\{1,2\}$, let $q_{i}$ be the neighbour of $w$ on $C_{i}$.

* If $q_{1}$ has degree 0 in $C_{2}$ (the case where $q_{2}$ has degree 0 in $C_{1}$ is symmetric), then let $C_{2}^{*}$ be induced by the edges in $E\left(C_{2}\right) \cup\left\{q_{1} w, w v\right\}$ and let $C_{1}^{*}$ be induced by the edges in $\left(E\left(C_{1}\right) \backslash\left\{q_{1} w\right\}\right) \cup\{u v\}$. Note that $C_{1}^{*}$ and $C_{2}^{*}$ are acyclic and have even size. Then $T_{1}^{*}=\left(T_{1} \backslash C_{1}\right) \cup C_{1}^{*}$ and $T_{2}^{*}=\left(T_{2} \backslash C_{2}\right) \cup C_{2}^{*}$ are good cacti.

* If $q_{1}$ has degree 2 in $C_{2}$ (the case where $q_{2}$ has degree 2 in $C_{1}$ is symmetric), then let $C_{2}^{*}$ be induced by the edges in $\left(E\left(C_{2}\right) \backslash\left\{q_{2} w\right\}\right) \cup\left\{q_{1} w\right\}$ and let $C_{1}^{*}$ be induced by the edges in $\left(E\left(C_{1}\right) \backslash\left\{q_{1} w\right\}\right) \cup\left\{q_{2} w, w v, v u\right\}$ (hence $C_{1}^{*}$ and $C_{2}^{*}$ are acyclic with one vertex, resp. $u$ and $q_{1}$, of degree 3$)$. Then $T_{1}^{*}=\left(T_{1} \backslash C_{1}\right) \cup C_{1}^{*}$ and $T_{2}^{*}=\left(T_{2} \backslash C_{2}\right) \cup C_{2}^{*}$ are good cacti.

* Finally, let us assume that $q_{1}$ has degree 1 in $C_{2}$ and that $q_{2}$ has degree 1 in $C_{1}$. This implies that $q_{1}=x_{2}$ and $q_{2}=x_{1}$. Then, let $C_{2}^{*}$ be induced by the edges in $\left(E\left(C_{2}\right) \backslash\left\{q_{2} w\right\}\right) \cup\left\{q_{1} w, v w, v u\right\}$ (since $C_{2}^{a}$ has odd length, this results in $C_{2}^{*}$ being an even-length cycle with a pending path) and let $C_{1}^{*}$ be induced by the edges in $\left(E\left(C_{1}\right) \backslash\left\{q_{1} w\right\}\right) \cup\left\{q_{2} w\right\}$ (hence, $C_{2}^{*}$ is an even-length path $\left(w, q_{2}=\right.$ $\left.\left.x_{1}, \ldots, u, \ldots, q_{1}\right)\right)$. Finally, $T_{1}^{*}=\left(T_{1} \backslash C_{1}\right) \cup C_{1}^{*}$ and $T_{2}^{*}=\left(T_{2} \backslash C_{2}\right) \cup C_{2}^{*}$ are good cacti.

We are now ready to prove our main result, the first part of Theorem 1.5.

Proof of Theorem 1.5. We prove the first part in the statement of Theorem 1.5 by proving that every connected $k$-degenerate graph $G$ with even size decomposes into at most $k$ good cacti, as they can each be further decomposed into at most three locally irregular subgraphs (Theorem 2.2). We prove this by induction on $|E(G)|$. The base case is when $G$ is a path of length 2 , in which case the claim obviously holds. Let us now focus on the general case.

Assume first that there exists $v \in V(G)$ such that $E(G)$ can be partitioned into two parts $A$ and $B$ of size at least 2 each, such that, for every $e \in A, f \in B$, if $e \cap f \neq \emptyset$ then $e \cap f=\{v\}$. In other words, $v$ is a cut-vertex of $G$ whose removal results in at least two connected components.

- If $A$ (and so $B$ ) has even size, then, by induction on $G[A]$ and $G[B]$, let $A=\bigcup_{j \leq k} T_{j}^{A}$ and $B=\bigcup_{j \leq k} T_{j}^{B}$ where each $T_{i}^{A}$ (resp., $T_{i}^{B}$ ) induces a (possibly empty) good cactus. Clearly, for every $1 \leq i \leq k, T_{i}=T_{i}^{A} \cup T_{i}^{B}$ induces a good cactus, and $E(G)=\bigcup_{i} T_{i}$.

- Otherwise, $|A|$ and $|B|$ are odd. Let $G_{A}$ (resp., $G_{B}$ ) be the graph induced by $A$ (resp., by $B$ ) with, in addition, a new pending edge $p$ incident to $v$. Since $|A|,|B| \geq 2, G_{A}$ and $G_{B}$ have strictly less edges than $G$ and we can proceed by induction. Let $A \cup\{p\}=\bigcup_{j \leq k} T_{j}^{A}$ and $B \cup\{p\}=\bigcup_{j \leq k} T_{j}^{B}$ where each $T_{i}^{A}$ (resp., $T_{i}^{B}$ ) induces a (possibly empty) good cactus. W.l.o.g., $p \in T_{1}^{A} \cap T_{1}^{B}$. For every $1<i \leq k$, let $T_{i}=T_{i}^{A} \cup T_{i}^{B}$ and let $T_{1}=\left(T_{1}^{A} \cup T_{1}^{B}\right) \backslash\{p\}$. For every $1 \leq i \leq k, T_{i}$ induces a good cactus, and $E=\bigcup_{i} T_{i}$.

Second, let us assume that there exists $v \in V(G)$ with degree $2 \leq d(v) \leq k+1$, incident to a vertex $u \in V(G)$ with degree 1 and such that $G[V(G) \backslash\{u, v\}]$ is connected. Let $w \in N(v) \backslash\{u\}$ and let $E^{\prime}=E(G) \backslash\{u v, v w\}$. Let $G^{\prime}=\left(V^{\prime}, E^{\prime}\right)$ where $V^{\prime}=V(G) \backslash\{u\}$ if $d(v)>2$ and 
$V^{\prime}=V(G) \backslash\{u, v\}$ otherwise. By the induction hypothesis on $G^{\prime}$, let $E^{\prime}=\bigcup_{j \leq k} T_{j}^{\prime}$ where each $T_{i}^{\prime}$ induces a (possibly empty) good cactus and, w.l.o.g., no edge of $T_{1}^{\prime}$ is incident to $v$ in $G^{\prime}$ (possible since $v$ has degree at most $k-1$ in $\left.G^{\prime}\right)$. Let $T_{j}=T_{j}^{\prime}$ if $j>1$ and let $T_{1}=T_{1}^{\prime} \cup\{u v, v w\}$. Then, $\left(T_{j}\right)_{1 \leq j \leq k}$ is a desired solution.

Now, if none of the previous two cases occurs, then, because $G$ is $k$-degenerate, we may assume that there is a vertex $v \in V(G)$ with $2 \leq d(v) \leq k$ and $G-v$ is connected. Let $u, w \in N(v)$ and let $G^{\prime}$ be the subgraph induced by $E(G) \backslash\{v u, v w\}$. Since $G^{\prime}$ is $k$-degenerate, connected and of even size $|E(G)|-2$, the induction hypothesis holds and there exists a family $\left(T_{1}, \ldots, T_{k}\right)$ of good cacti decomposing $G^{\prime}$. Since $v$ has degree at most $k-2$ in $G^{\prime}$, there are two good cacti, say $T_{1}$ and $T_{2}$, such that $v$ is not incident to any edge of $E\left(T_{1}\right) \cup E\left(T_{2}\right)$. All conditions are now met to apply Lemma 3.1, which ensures that the graph induced by the edges $E\left(T_{1}\right) \cup E\left(T_{2}\right) \cup\{u v, u w\}$ can be decomposed into two good cacti $T_{1}^{*}, T_{2}^{*}$, hence yielding a desired decomposition $\left(T_{1}^{*}, T_{2}^{*}, T_{3}, \ldots, T_{k}\right)$ of $G$ into $k$ good cacti.

\section{Improved bounds for particular classes of degenerate graphs}

Throughout this section, we improve the bound in Theorem 1.5 for two classes of degenerate graphs, namely $k$-trees and planar graphs.

\section{1. $k$-trees}

Let $k \geq 2$ be an integer. A $k$-tree is any graph built recursively as follows. A complete graph with $k+1$ vertices is a $k$-tree. Given a $k$-tree and a complete subgraph $C$ of order $k$ in it, a new $k$-tree is obtained by adding a new vertex adjacent to every vertex of $C$. Note that $k$-trees are $k$-degenerate. Graphs of treewidth at most $k$ are precisely the subgraphs of $k$-trees.

To any $k$-tree $G$ may be associated an ordering of its vertices, called building order, corresponding to the order in which they have been added to create $G$ (with the initial clique as prefix).

Claim 4.1. Let $G$ be any $k$-tree and $H$ be any subgraph of $G$ that is a $k$-tree. Then there exists a building order of $G$ with the vertices of $H$ as prefix.

Proof. The proof is by induction on the number $n$ of vertices of $G$. The result is obvious if $n=k+1$. Let $H$ be a subgraph of $G$ that is a $k$-tree. If $H \neq G$ (otherwise the result is obvious), then there is a vertex $v$ of degree $k$ in $V(G) \backslash V(H)$. Indeed, for purpose of contradiction, let us assume that all vertices in $V(G) \backslash V(H)$ have degree larger than $k$. Let us consider any building order $\mathcal{B}$ of $G$ and let $v$ be the last vertex of $V(G) \backslash V(H)$ in this order. Since $v$ has degree larger than $k$ in $G$, there must be a vertex $w \in V(H)$, added after $v$ in $\mathcal{B}$, and such that the neighbourhood $N$ of $w$ (when it is added) contains $v$. Note that $|N|=k$. Consider any component $C$ of $G-N$ not containing $w$ : it must contain a vertex $y$ with degree $k$ in $G$ (among the vertices of $C$ farthest from $N$, the one that has been added last must have degree $k$ ). Hence, $y \in V(H)$. Therefore, $N \cap V(H)$ is a $y, w$-separator in $H$ of size strictly less than $k$, contradicting the fact that $H$ is a $k$-tree.

To conclude, the induction hypothesis can be applied to $G^{\prime}=G-v$ (which is a $k$-tree) to obtain a building order $\mathcal{O}$ of $G^{\prime}$ with $V(H)$ as prefix. The desired building order of $G$ is obtained by adding $v$ at the end of $\mathcal{O}$.

We are now ready to improve Theorem 1.5 for $k$-trees, which states that their irregular chromatic index is at most $3 k+1$. We treat the cases $k=2$ and $k \geq 3$ separately.

Theorem 4.2. For every decomposable 2 -tree $G$, we have $\chi_{\text {irr }}^{\prime}(G) \leq 4$.

Proof. The fact that $G$ is decomposable implies that $G$ has at least 4 vertices. It is easy to see that the result holds if $G$ has 4 or 5 vertices (there are only three graphs as $G$ in this case, as shown in the left part of Figure 1). Hence, let us assume that $G$ has at least 6 vertices.

First, let us point out two pathological cases where a decomposition of $G$ in at most two locally irregular subgraphs can easily be constructed. On the one hand, if $G$ is a fan, i.e., a path (called external path) plus a universal vertex, with at least seven vertices, then consider the decomposition, depicted in Figure 2 (D), into two spiders where the center has degree at least 3. If $G$ is a fan with exactly six vertices, then consider the decomposition depicted in Figure 2 (D'). If $G$ is a book, i.e., 


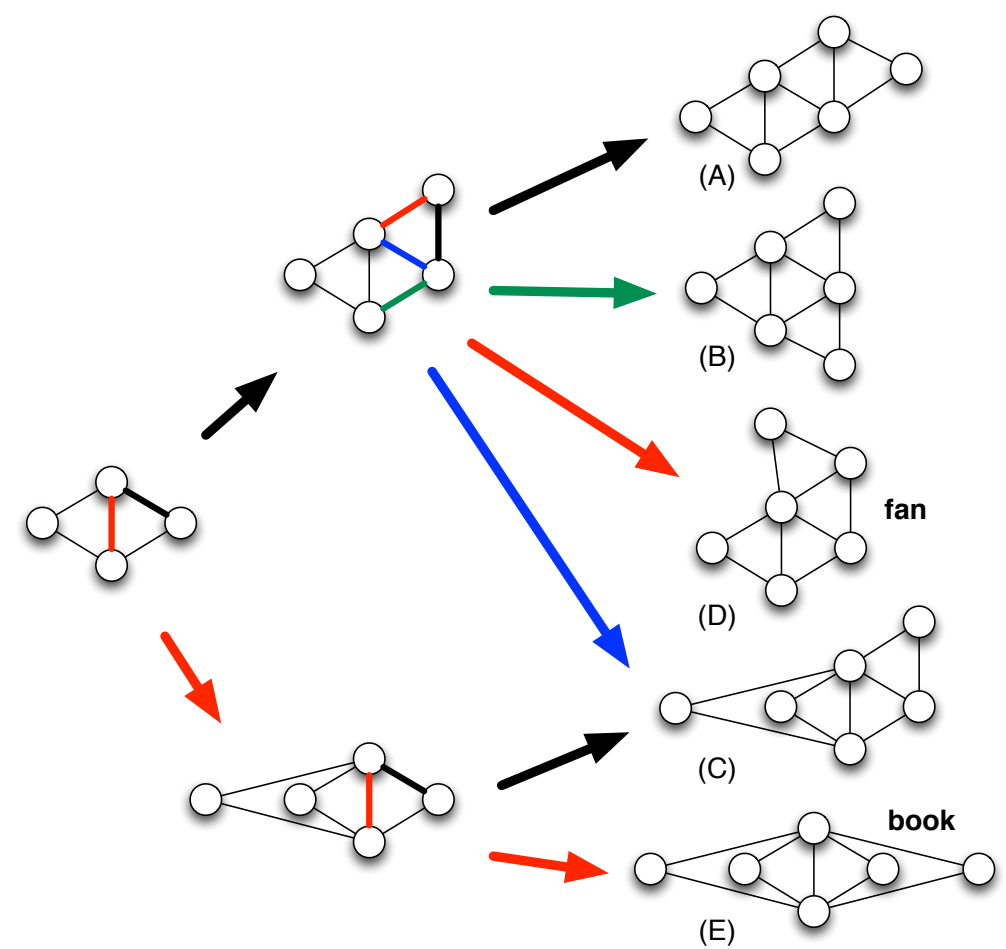

Figure 1: Generation of all non-isomorphic 2-trees on 6 vertices, starting from the single 2-tree on 4 vertices. An arrow coloured with colour $x$ indicates that the new vertex has been made adjacent to the edge colored with colour $x$.

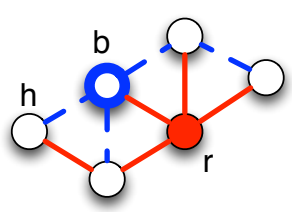

(A)

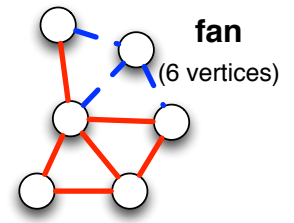

(D')

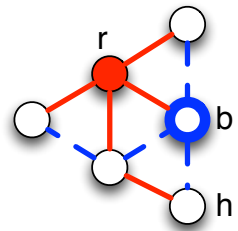

(B)

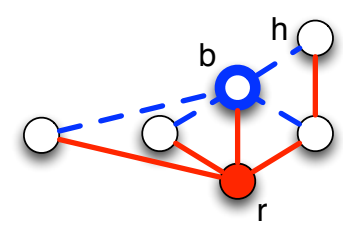

(C)

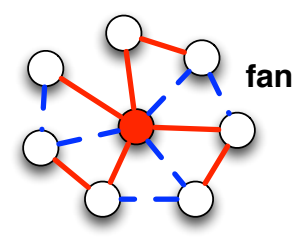

(D)

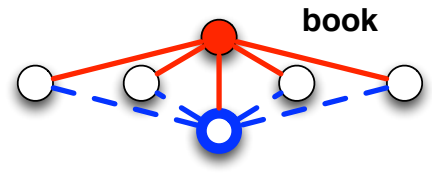

(E)

Figure 2: Initial decompositions of the 2-trees (A), (B), (C), and schematic representations of the decompositions of (D) and (E). In all cases, one part is depicted in dotted edges and the second part is in solid line. In both cases (D) (fan with at least 7 vertices), (D') (fan with 6 vertices) and (E) (book), the two parts induce locally irregular graphs.

a 2 -tree with order $n$ and $n-2$ vertices with degree 2 , then consider the decomposition, given in Figure $2(\mathrm{E})$, into two stars where the center has degree at least 3. From now on, we can thus assume that $G$ is not a fan nor a book.

As shown in Figure 1, there are exactly five non-isomorphic 2-trees with 6 vertices, including one fan and one book. Since $G$ is not a fan nor a book, it must contain some of the three graphs (A), (B), (C) (on Figure 1) as a subgraph. Indeed, let us consider a building order of $G$. If its 
prefix (of length 6) induces one of the graphs (A), (B), (C), then we are done. Otherwise, there are two cases to be considered.

- If its prefix (of length 6) induces a fan, then let us consider the largest prefix of it that induces a fan and let $v$ be the next vertex in the order (that vertex must exist since $G$ is not a fan). If $v$ is made adjacent to both ends of an edge of the external path, then this creates a subgraph (A), while it creates a subgraph $(\mathrm{C})$ otherwise.

- If its prefix (of length 6) induces a book, then let us consider the largest prefix of it that induces a book and let $v$ be the next vertex in the order (that vertex must exist since $G$ is not a book). Adding $v$ then creates a subgraph (C).

Let $v_{1}, \ldots, v_{n}$ be a building order of $G$. From the previous paragraph and Claim 4.1, we may assume that $n \geq 6$ and $\left\{v_{1}, \ldots, v_{6}\right\}$ induces either of (A), (B), (C). We consider the three cases simultaneously in what follows. In each of the three cases, let us define the vertices $r, b, h$ as depicted in Figure 2.

By induction on $6 \leq i$, we prove that, for every $6 \leq i \leq n$, the edge set of $G_{i}=G\left[\left\{v_{1}, \ldots, v_{i}\right\}\right]$ can be partitioned into two sets $X_{i}$ and $Y_{i}$, both inducing a tree containing a vertex with degree at least 3 that is adjacent to some leaf. For the base case $i=6$, the set $X_{i}$ is depicted with solid red edges in Figure 2 and $Y_{i}$ is depicted with dotted blue edges in Figure 2. More generally, we prove that, for all $6 \leq i \leq n, E\left(G_{i}\right)$ can be partitioned into $X_{i}$ and $Y_{i}$ both inducing a tree and such that:

1. vertex $r$ has degree at least 4 in $G\left[X_{i}\right]$;

2. vertex $b$ has degree 1 in $G\left[X_{i}\right]$ and degree at least 3 in $G\left[Y_{i}\right]$;

3. vertex $h$ has degree 1 in $G\left[Y_{i}\right]$; and

4. every vertex but $r$ has degree at least 1 in both $G\left[X_{i}\right]$ and $G\left[Y_{i}\right]$.

The result holds for $|V(G)|=6$ by defining $X_{6}$ and $Y_{6}$ as in Figure 2. Now, let us assume that $X_{i-1}$ and $Y_{i-1}$ partition $E\left(G_{i-1}\right)$ and satisfy the desired properties. Let $v_{i}$ be added as neighbour of $u, w \in V\left(G_{i-1}\right)$, where $u w \in E\left(G_{i-1}\right)$. If $b \in\{u, w\}$, then set $b=w$. If $r$ (resp., $h$ ) belongs to $\{u, w\}$ (note that they cannot both belong to $\{u, w\}$ since $u w \in E(G)$ and $r h \notin E(G)$ ), then set $u=r$ (resp., $u=h$ ). Then, add $v u$ to $X_{i-1}$, i.e., $X_{i}=X_{i-1} \cup\{u v\}$, and $v_{i} w$ to $Y_{i-1}$, i.e., $Y_{i}=Y_{i-1} \cup\left\{v_{i} w\right\}$. It is easy to see that all four properties still hold. Moreover, by the fourth property, $X_{i}$ and $Y_{i}$ induce trees with some vertex with degree at least 3 that is adjacent to some leaf.

Hence, $E(G)$ can be partitioned into two sets $X$ and $Y$ such that each of $G[X]$ and $G[Y]$ induces a tree containing a vertex with degree at least 3 that is adjacent to some leaf. Any tree $T$ with this property has $\chi_{\text {irr }}^{\prime}(T)=2$, recall Theorem 2.1. The result then follows. That is, $X$ (resp., $Y$ ) can be partitioned into $X^{1}, X^{2}$ (resp., $Y^{1}, Y^{2}$ ), with each of $G\left[X^{1}\right], G\left[X^{2}\right], G\left[Y^{1}\right]$ and $G\left[Y^{2}\right]$ being locally irregular. Hence, $\chi_{\text {irr }}^{\prime}(G) \leq 4$.

Theorem 4.3. For every $k$-tree $G$ with $k \geq 3$, we have $\chi_{\text {irr }}^{\prime}(G) \leq 2 k$.

Proof. This proof is similar to that of Theorem 4.2 except that it is simpler due to a simpler base case. If $G$ is reduced to a clique, then the result follows from [2]. Hence, let us assume that $G$ has $n>k+1$ vertices. We prove that $E(G)$ can be partitioned into $k$ parts $X_{1}, \ldots, X_{k}$ such that, for every $1 \leq i \leq k$, set $X_{i}$ induces a tree with a vertex with degree at least 3 that is adjacent to some leaf. Any tree $T$ with this property has $\chi_{\text {irr }}^{\prime}(T)=2$ (Theorem 2.1), and so the theorem follows.

The proof is by induction on $|V(G)|>k+1$. Let us consider a building order of $G$. Let $K$ be the complete graph induced by the $k+1$ first vertices, and let us denote by $v_{1}, \ldots, v_{k}, a, b$ the $k+2$ first vertices of the order. If $|V(G)|=k+2$, then let $X_{1}=\left\{v_{1} a, v_{1} b\right\} \cup \bigcup_{1<j<k}\left\{v_{1} v_{j}\right\}$, $X_{i}=\left\{v_{i} a, v_{i} b\right\} \cup \bigcup_{i<j \leq k}\left\{v_{i} v_{j}\right\}$ for $1<i<k$ and $X_{k}=\left\{v_{k} a, v_{k} b, v_{k} v_{1}\right\}$. Clearly, $E(G)=\bigcup_{i \leq k} X_{i}$, each $X_{i}$ induces a tree, and for every $1 \leq i \leq k$, vertex $v_{i}$ has degree at least 3 in $X_{i}$ and vertex $v_{i+1}$ (resp., $v_{1}$ for $i=k$ ) is a neighbour of degree 1 of $v_{i}$ in $X_{i}$. Finally, every vertex but those in $\left\{v_{1}, \ldots, v_{k}\right\}$ is adjacent to some edge in $X_{i}$ for every $1 \leq i \leq k$. 
Then, each time a new vertex $v$ is added, exactly one of its $k$ incident edges is added to $X_{i}$ for every $1 \leq i \leq k$ with the only constraint that, for every $1 \leq i \leq k$, if $v v_{i} \in E(G)$, then $v v_{i}$ is added to $X_{i}$. It is easy to check that all desired properties are preserved.

\subsection{Planar graphs}

Before proceeding with the proof of the main result in this section, let us introduce a few new and existing tools. We first need the notion of light edges in planar graphs. An $x$-vertex refers to a vertex with degree precisely $x$. By an $(x, y)$-edge, we mean an edge whose one end is an $x$-vertex and other end is a $y$-vertex.

Recall that a planar graph is a graph embedded in the plane in such a way that no two edges cross. It is a well-known fact (from Euler's formula), that planar graphs are 5-degenerate. In planar graphs with minimum degree 5, even lighter structural properties can actually be established.

Theorem 4.4 (see e.g. [5], Theorem 3.1). Every planar graph with minimum degree 5 has a $(5,5)$ edge or $(5,6)$-edge.

In what follows, whenever referring to a light edge we will thus mean a $(5,5)$-edge or a $(5,6)$ edge.

We will also be needing the following counterpart of Lemma 3.1 when three edges incident to a same vertex remain to be added to good cacti.

Lemma 4.5. Let $G$ be a graph, and let $T$ be a subgraph of $G$ that is a good cactus not containing a vertex $v \in V(G)$. Then, for every three edges vu,vw, vx incident to $v$, there exists a subgraph $T^{*}$ of $G$ that is a good cactus with $E\left(T^{*}\right)=E(T) \cup\{v u, v w, v x\}$ or $E\left(T^{*}\right)=E(T) \cup\{e\}$ for some $e \in\{v u, v w, v x\}$.

Proof. If adding all three of $v u, v w, v x$ to $T$ leads to a good cactus, then we are done. Otherwise, this means that at least two of $u, w, x$, say $u, w$ w.l.o.g., belong to the same connected component of $T$. If adding $v u$ to $T$ is not correct, then this means that this creates an odd-length path in $T$, which is possible only when $u$ is an end of an even-length path $P$ of $T$. By the same argument, $w$ is the second end of $P$. Now, if $P$ goes through $x$ (meaning $x$ is an internal vertex of $P$ ), then adding $v x$ to $T$ yields the correct conclusion, as $P$ would become a tree with a 3 -vertex. Thus, we may lastly assume that $x$ does not belong to $P$, in which case adding all three of $v u, v w, v x$ to $T$ is actually correct: as a result, $P$ becomes an even-length cycle with the edge $u x$ attached, possibly joining the cycle to another connected component of $T$.

We are now ready to prove the main result in this section. Since every planar graph is 5degenerate, from Theorem 1.5 we get that every decomposable planar graph $G$ verifies $\chi_{\text {irr }}^{\prime}(G) \leq 16$. We slightly improve this bound down to 15 .

Theorem 4.6. For every connected planar graph $G$ with even size, we have $\chi_{\mathrm{irr}}^{\prime}(G) \leq 14$. Consequently, by Theorem 1.3, for every decomposable planar graph $G$ we have $\chi_{\mathrm{irr}}^{\prime}(G) \leq 15$.

Proof. We prove the claim by proving that every connected planar graph $G$ with even size decomposes into four good cacti $T_{1}, \ldots, T_{4}$ and one even forest $T_{5}$, i.e., a forest whose all trees have even size. This implies the result, as good cacti have irregular chromatic index at most 3 (Theorem 2.2) and even forests have irregular chromatic index at most 2 (Theorem 2.1). This is proved by induction on $|E(G)|$. Since this is trivially true when $|E(G)|=2$, we focus on the general case. This is done by proving gradually that the claim holds under certain circumstances, until we get to the point where the claim is ultimately proved to hold in general.

We start off by considering bridges. Assume that $G$ has a bridge $u v$, and let $G_{u}$ (resp. $G_{v}$ ) denote the connected component of $G-u v$ that contains $u$ (resp. $v$ ). Since $G$ has even size, w.l.o.g. $G_{u}$ has even size while $G_{v}$ has odd size. Assume $\left|E\left(G_{u}\right)\right| \geq 2$. Then both $G_{u}$ and $G_{v}+u v$ are planar graphs with even size smaller than that of $G$, so they admit desired decompositions $\left(T_{1}, \ldots, T_{5}\right)$ and $\left(T_{1}^{\prime}, \ldots, T_{5}^{\prime}\right)$, respectively. Since $G_{u}$ and $G_{v}+u v$ intersect only in $u$, we get that $\left(T_{1} \cup T_{1}^{\prime}, \ldots, T_{5} \cup T_{5}^{\prime}\right)$ is a desired decomposition of $G$, since identifying two vertices from two disjoint good cacti clearly results in a good cactus, and similarly for two even forests. So we may now assume that $G$ contains no such bridge, which we call good bridges below. Note that a bridge is not good if and only if one of its end is a 1-vertex. 
Claim 4.7. We may assume that $G$ has no good bridge.

Since $G$ is planar, it has minimum degree at most 5. In some cases, assuming the existence in $G$ of vertices with small degree, the induction hypothesis can be invoked on a subgraph $G^{\prime}$ of $G$ to deduce a decomposition that can be extended to $G$. More precisely:

Claim 4.8. We may assume that $G$ does not have a 1-vertex adjacent to a vertex of degree at most 6 .

Proof. Let us assume $G$ has a 1-vertex $u$ that is adjacent to a vertex $v$ of degree at most 6 . Let us denote by $w_{1}, \ldots, w_{d}$ the $d \leq 5$ neighbours of $v$ different from $u$. If one edge $v w_{i}$ is such that $G^{\prime}=G-u-\left\{v w_{i}\right\}$ is connected, then we can deduce a decomposition $\left(T_{1}, \ldots, T_{5}\right)$ of $G^{\prime}$ by induction, and extend it to $G$ by adding $u v, v w_{i}$ to one of $T_{1}, \ldots, T_{5}$ that does not contain $v$ (one such exists since $v$ has degree at most 4 in $G^{\prime}$ ). This results in a correct decomposition of $G$, as attaching a pendant path of length 2 to a good cactus or an even forest, respectively, results in a good cactus or an even forest, respectively.

So assume that $G-u-\left\{v w_{i}\right\}$ is disconnected for every $i$, i.e., $v w_{i}$ is a bridge for every $i$. Since $G$ has even size, note that it cannot be that the connected component that contains $w_{i}$ in $G-v w_{i}$ has odd size for every $i$. Let us thus assume that the connected component that contains $w_{1}$ in $G-v w_{1}$ has even size. Recall that this connected component cannot have size at least 2 , as otherwise $G$ would have a good bridge. Thus, by Claim 4.7, $w_{1}$ is a 1 -vertex. In that case, we consider $G^{\prime}=G-u-w_{1}$, which is planar of even size smaller than that of $G$, and a decomposition $\left(T_{1}, \ldots, T_{5}\right)$ of $G^{\prime}$. Clearly, adding $u v, v w_{1}$ to any of $T_{1}, \ldots, T_{5}$ yields a desired decomposition of $G$.

Claim 4.9. We may assume that, for every $x \in\{2,3,4\}, G$ has no $x$-vertex.

Proof. Let us consider the three cases depending on $x \in\{2,3,4\}$.

- Assume $G$ has a 2-vertex $v$ with neighbours $u_{1}, u_{2}$.

- If $G^{\prime}=G-v$ is connected, then, because it has even size, $G^{\prime}$ admits a decomposition $\left(T_{1}, \ldots, T_{5}\right)$ by induction. Now, since none of the good cacti $T_{1}, T_{2}$ contains $v$, using Lemma 3.1 we can deduce a desired decomposition $\left(T_{1}^{*}, T_{2}^{*}, T_{3}, \ldots, T_{5}\right)$ of $G$.

- Otherwise, if $G-v$ is not connected, then both edges adjacent to $v$ are bridges in $G$. Since $v$ cannot be adjacent to a 1 -vertex and none of $v u_{1}, v u_{2}$ is a good bridge, we get a contradiction.

From now on, we can thus assume that $G$ has no 2 -vertex.

- Assume $G$ has a 3 -vertex $v$ with neighbours $u_{1}, u_{2}, u_{3}$.

- If $G^{\prime}=G-\left\{v u_{i}, v u_{j}\right\}$ is connected for any two edges $v u_{i}, v u_{j}$, then, because it has even size, $G^{\prime}$ admits a decomposition $\left(T_{1}, \ldots, T_{5}\right)$ by induction. At least two of the four good cacti $T_{1}, \ldots, T_{4}$, say $T_{1}, T_{2}$ without loss of generality, do not contain $v$. Thus, using Lemma 3.1 we can deduce a desired decomposition $\left(T_{1}^{*}, T_{2}^{*}, T_{3}, \ldots, T_{5}\right)$ of $G$.

- Thus, we may suppose that $G-\left\{v u_{1}, v u_{2}\right\}$ is disconnected, which means that $G-v u_{3}$ is disconnected as well. In other words, $v u_{3}$ is a bridge of $G$. Since $u_{3}$ cannot be a 1-vertex (because $v$ has degree 3 and by Claim 4.8), we get that $v u_{3}$ is actually a good bridge, which is impossible.

Thus, we may now suppose that $G$ has no 3 -vertex.

- Assume $G$ has a 4 -vertex $v$ with neighbours $u_{1}, u_{2}, u_{3}, u_{4}$.

- If $G-\left\{v u_{i}, v u_{j}\right\}$ is connected for any two edges $v u_{i}, v u_{j}$, then Lemma 3.1 can again be applied to deduce a desired decomposition of $G$ similarly as in the previous case. 
- Thus, we may suppose that $G-\left\{v u_{1}, v u_{2}\right\}$ is disconnected. Since $v$ cannot be adjacent to a 1-vertex (by Claim 4.8), none of $v u_{3}, v u_{4}$ can be a bridge, as otherwise it would be a good bridge, a contradiction. However, $G-\left\{v u_{3}, v u_{4}\right\}$ is disconnected as well. Thus, $G-v$ has two connected components, one of which contains $u_{1}, u_{2}$ while the second one contains $u_{3}, u_{4}$. Then it can be noticed that $G-\left\{v u_{1}, v u_{3}\right\}$ is connected, a case we have already treated.

This concludes the proof of the claim.

Thus, we may now suppose that $G$ has no $x$-vertex for $x=2,3,4$. Furthermore, all 1-vertices of $G$ are adjacent to vertices with degree at least 7, and, by arguments used to deal with 1-vertices above (end of the proof of Claim 4.8), we can also assume that $G$ has no two 1-vertices adjacent to a same vertex. These arguments imply that $G^{-}$, the graph obtained from $G$ by removing all 1-vertices, is a planar graph of minimum degree exactly 5 . By Theorem 4.4, it has a light edge $u v$, which is either a $(5,5)$-edge or a $(5,6)$-edge. Back in $G$, by all the previous properties, we deduce that $u v$ is either a $(5,5)$-edge (type $\mathbf{A})$ or a $(5,6)$-edge where none of $u, v$ is adjacent to a 1 -vertex (type $\mathbf{B}$ ), or a $(5,7)$-edge where the one of $u, v$ with degree 7 is adjacent to a 1-vertex (type $\mathbf{C}$ ). In what follows, we always implicitly assume that $d(u) \leq d(v)$.

Assume first that $G$ has a type-C edge $u v$. Let us denote by $w$ the 1 -vertex adjacent to $v$. Since $G$ has no good bridge (Claim 4.7), note that $G^{\prime}=G-w-\{u v\}$ is connected. Since $G^{\prime}$ is a planar graph with even size smaller than that of $G$, it admits a decomposition $\left(T_{1}, \ldots, T_{5}\right)$. By that decomposition, there is a $T_{i}$ of $T_{1}, \ldots, T_{5}$ that does not contain $u$, since this vertex has degree 4 in $G^{\prime}$. Then adding $u v, v w$ to $T_{i}$ (which either adds a connected component being a path of length 2 , or two pendant vertices attached to a same vertex) results in a good cactus if $T_{i}$ was one, or in an even forest if $T_{i}$ was one. We thus get a desired decomposition of $G$.

To deal with $u v$ being type $\mathrm{A}$ or $\mathrm{B}$, let us focus on 5 -vertices and 6 -vertices that are cut-vertices in $G$.

Claim 4.10. We may assume that $G$ has no 5-vertex or 6-vertex being a cut-vertex.

Proof. Assume that $G$ has a 5 -vertex or 6 -vertex $x$ that is a cut-vertex. Since $x$ cannot be adjacent to a 1-vertex, and we have assumed that $G$ has no good bridge (Claim 4.7), we may assume that $x$ is incident to no bridge at all. From this, we deduce that $G-x$ has at most three connected components $C_{1}, C_{2}, C_{3}$, with $C_{3}$ possibly empty. Let $x y_{1}$ and $x y_{2}$ be edges incident to $x$ where $y_{1}$ is in $C_{1}$ while $y_{2}$ is in $C_{2}$. Then $G^{\prime}=G-\left\{x y_{1}, x y_{2}\right\}$ is a connected planar graph with even size smaller than that of $G$, which admits a good decomposition $\left(T_{1}, \ldots, T_{5}\right)$ by the induction hypothesis. We can now consider one $T_{i}$ not containing $x$ (which exists since $x$ has degree 3 in $G^{\prime}$ ), and add $x y_{1}, x y_{2}$ to $T_{i}$, yielding either a good cactus (if $T_{i}$ was one), or an even forest (otherwise). This is because joining two good cacti by a path of length 2 yields a good cactus, and similarly for even forests.

Thus we may now assume that $G$ has no 5 -vertex being a cut-vertex. Assume now that, in $G$, $u v$ is a light edge of type A. Let us denote by $w_{1}, \ldots, w_{4}$ the neighbours of $u$ different from $v$. By the above, we may assume that $G^{\prime}=G-\left\{u v, u w_{1}, u w_{2}, u w_{3}\right\}$ is a connected planar graph with even size smaller than that of $G$. There thus exists a good decomposition $\left(T_{1}, \ldots, T_{5}\right)$ of $G^{\prime}$. Since $v$ has degree 4 in $G^{\prime}$, there is one $T_{i}$ of $T_{1}, \ldots, T_{5}$ that does not contain $v$. We consider two cases.

- $i \neq 5$. That is, $T_{i}$ is a good cactus. If $u w_{4}$ does not belong to $T_{i}$, then adding $w_{1} u, u v$ to $T_{i}$ results in a good cactus. We are then left with treating the two edges $w_{2} u, w_{3} u$, which can be added, via Lemma 3.1 to two of $T_{1}, \ldots, T_{4}$ that are not $T_{i}$ and do not contain $w_{4} u$.

So now assume that $u w_{4}$ belongs to $T_{i}$. If adding $u v$ to $T_{i}$ does not result in a component of $T_{i}$ becoming an odd-length path, then $T_{i}$ remains a good cactus. Then $w_{1} u, w_{2} u, w_{3} u$ remain to be treated, and there is so far only one, $T_{i}$, of $T_{1}, T_{2}, T_{3}, T_{4}$ containing $v$. Assume $T_{i}=T_{4}$ without loss of generality. Now, using Lemma 4.5 either three (in which case we are done) of $w_{1} u, w_{2} u, w_{3} u$ can be added to $T_{1}, T_{2}, T_{3}$ correctly, or only one of them, say $w_{1} u$ to $T_{3}$. Lemma 3.1 can now be used to add $w_{2} u, w_{3} u$ to $T_{1}, T_{2}$, resulting in a desired decomposition of $G$. 
So lastly assume that adding $u v$ does result in a component of $T_{i}$ becoming an odd-length path. Then $T_{i}$ has a connected component being an even-length path $P$ whose one end is $u$ (and $P$ reaches $u$ via $w_{4}$ ). If, say, $w_{1}$, does not belong to $P$, then, by adding both $w_{1} u, u v$ to $T_{i}$, part $T_{i}$ remains a good cactus (in particular because $P$ gets added into a subgraph with maximum degree at least 3). Then $w_{2} u, w_{3} u$ can again be treated using Lemma 3.1. So we may assume that all of $w_{1}, w_{2}, w_{3}$ belong to $P$, which means that at least two of $w_{1}, w_{2}, w_{3}$ are internal vertices of $P$, one of which is not adjacent to $w_{4}$ in $P$. Assume $w_{1}$ is an internal vertex of $P$ not adjacent to $w_{4}$. We here remove $w_{4} u$ from $T_{i}$, and add $w_{1} u, u v$ to $T_{i}$. This way, in $T_{i}$, the component $P$ becomes a tree in which $w_{1}$ has degree 3 . It now remains to deal with $w_{2} u, w_{3} u, w_{4} u$, while $u$ so far belongs to only one good cactus, $T_{i}$. Using Lemmas 3.1 and 4.5, we can eventually do so, and thus construct a desired decomposition of $G$.

- $i=5$. That is, $T_{i}$ is the even forest. Again, if $w_{4} u$ does not belong to $T_{i}$, then we are done as earlier. So we may assume that $w_{4} u$ belongs to $T_{i}$. Then there are only four remaining edges to be treated $\left(w_{1} u, w_{2} u, w_{3} u, u v\right)$, all incident to $u$, while $u$, this far, does not belong to any of $T_{1}, \ldots, T_{4}$. We can here complete the decomposition by applying Lemma 3.1 twice.

We are now left with the case where $u v$ is a type-B edge. First of all, by Claim 4.10, we can assume that $v$ is not a cut-vertex. Thus, we can assume that $G-v$ is connected. Since $u$ has degree 4 in $G-v$, the graph $G^{\prime}=G-u-v$ has at most four connected components $C_{1}, \ldots, C_{d}$. Furthermore, in $G$, for each $C_{i}$, each of $u, v$ has at least one neighbour in $C_{i}$ (since $v$ is not a cut-vertex). To finish off the proof, we deal with the number $d$ of $C_{i}$ 's, and the connection between these components and $u, v$ in $G$. We denote by $x_{1}, \ldots, x_{4}$ the four neighbours of $u$ different from $v$, and by $y_{1}, \ldots, y_{5}$ the five neighbours of $v$ different from $u$.

If $d=1$, i.e., $G^{\prime}$ is connected, then note that $G^{\prime}$ is a planar graph with even size $|E(G)|-10$. It thus admits a decomposition $\left(T_{1}, \ldots, T_{5}\right)$ by the induction hypothesis. We extend it to $G$ as follows. First, we add $u v, v y_{5}$ to $T_{5}$, which adds a path of length 2 which is either pendant or isolated in the even forest. To eventually add the eight remaining edges $x_{1} u, x_{2} u, x_{3} u, x_{4} u$ and $y_{1} v, y_{2} v, y_{3} v, y_{4}, v$ to the decomposition, we just apply Lemma 3.1 four times: twice on $u$, and twice on $v$.

Thus, we may now suppose that $G^{\prime}$ is not connected, i.e., $d \neq 1$. Note that $G^{\prime \prime}=G-v$ is connected and has even size. Thus, by the induction hypothesis, $G^{\prime \prime}$ has a good decomposition $\left(T_{1}, \ldots, T_{5}\right)$. Since $u$ has degree 4 in $G^{\prime \prime}$, one of $T_{1}, \ldots, T_{5}$ does not contain $u$.

- If $T_{5}$ does not contain $u$, then we add $u v$ and $v y_{1}$ to $T_{5}$. Note that this adds a path of length 2 that is pendant or isolated in $T_{5}$, so it remains an even forest. It remains to add the edges $v y_{2}, v y_{3}, v y_{4}, v y_{5}$ to the decomposition, which can be added to some of $T_{1}, \ldots, T_{4}$ by employing Lemma 3.1 twice on $v$.

- Now assume $T_{5}$ contains $u$. Then, one of $T_{1}, \ldots, T_{4}$, say $T_{1}$ w.l.o.g., does not contain $u$. Since $d \geq 2$, there are $y_{i}, y_{j}$ that belong to different connected components of $G^{\prime}$. Then we add $u v, v y_{i}, v y_{j}$ to $T_{1}$. Note that $T_{1}$ remains a good cactus, since the three edges we have added are not involved in any cycle of $T_{1}$. Furthermore, these edges belong to a connected component with maximum degree at least 3 , which is thus not an odd-length path. It remains three edges incident to $v$ to be added to the decomposition, while only $T_{1}$ contains $v$ so far. The three edges can then be added to $T_{2}, T_{3}, T_{4}$ using Lemma 4.5, and then Lemma 3.1 if needed.

\section{Conclusion}

In this work, we have improved known upper bounds on the irregular chromatic index of some families of degenerate graphs. Similarly as for other existing proofs, our proofs involve decompositions into auxiliary structures that are to be further decomposed. As a consequence, most known bounds, including ours, are still far from the conjectured one in Conjecture 1.1, even for very particular classes of graphs. Still, we believe that the decomposition methods we have introduced in this work can lead to more progress towards the conjecture. 
A prime line of research for future work would thus be to aim at lowering those bounds further. As a first appealing case, we believe the case of 2-degenerate graphs is of interest, as many colouring problems tend to become easy for those graphs. Also, it is worth recalling that there is an intricate connection between 2-degenerate graphs and exceptional graphs, as all exceptional graphs are 2degenerate. Planar graphs also sound interesting to investigate further for the same reasons, and because our bound in Theorem 4.6 leaves more space for improvement. A good compromise could be to consider outerplanar graphs, which are both 2-degenerate and planar. Improving our bound of 7 for these graphs would be an interesting first step.

Our result on 2-trees (Theorem 4.2) is also interesting in that regard, as 2-trees are 2-degenerate. We did not manage to come up with a proof of Conjecture 1.1 for these graphs, which might look surprising due to their very specific structure. Perhaps a way to progress towards all these concerns could be to consider the class of maximal outerplanar graphs, which form a subclass of 2-trees.

\section{References}

[1] O. Baudon, J. Bensmail, H. Hocquard, M. Senhaji, É. Sopena. On locally irregular decompositions of subcubic graphs. Opuscula Mathematica, 38(6):795-817, 2018.

[2] O. Baudon, J. Bensmail, J. Przybyło, and M. Woźniak. On decomposing regular graphs into locally irregular subgraphs. European Journal of Combinatorics, 49:90-104, 2015.

[3] O. Baudon, J. Bensmail, and É. Sopena. On the complexity of determining the irregular chromatic index of a graph. Journal of Discrete Algorithms, 30:113-127, 2015.

[4] J. Bensmail, M. Merker, and C. Thomassen. Decomposing graphs into a constant number of locally irregular subgraphs. European Journal of Combinatorics, 60:124-134, 2017.

[5] S. Jendrol', and H.-J. Voss. Light subgraphs of graphs embedded in the plane - A survey. Discrete Mathematics, 313:406-421, 2013.

[6] M. Karoński, T. Łuczak, and A. Thomason. Edge weights and vertex colours. Journal of Combinatorial Theory, Series B, 91:151-157, 2004.

[7] C.N. Lintzmayer, G.O. Mota, M. Sambinelli. Decomposing split graphs into locally irregular graphs. Preprint arXiv:1902.00986.

[8] B. Lužar, J. Przybyło, and R. Soták. New bounds for locally irregular chromatic index of bipartite and subcubic graphs. Journal of Combinatorial Optimization, 36(4):1425-1438, 2018.

[9] J. Przybyło. On decomposing graphs of large minimum degree into locally irregular subgraphs. Electronic Journal of Combinatorics, 23(2):\#P2.31, 2016. 